Check for updates

Cite this: RSC Adv., 2022, 12, 4072

\title{
Novel urea derivative-loaded PLGA nanoparticles to inhibit caries-associated Streptococcus mutans
}

\begin{abstract}
Mengyun Zhang, $\mathbb{D} \dagger^{\mathrm{a}}$ Ying Liao, $\dagger^{\mathrm{b}}$ Xin Tong ${ }^{* \mathrm{c}}$ and Fuhua Yan (D) *d
Caries is the most common chronic infectious disease in the human oral cavity and the existing anti-caries agents may lead to drug resistance and microecological imbalance. A novel urea derivative, 1,3-bis[3,5bis(trifluoromethyl)phenyl]urea, has a potentially prominent antibacterial effect on cariogenic bacterial strain Streptococcus mutans UA159. In this study, we encapsulated the water-insoluble urea derivative in poly(lactic-co-glycolic acid) (PLGA) nanoparticles, performed physicochemical characterizations and explored its potential as a caries-preventive agent. The results showed that the drug-loaded PLGA nanoparticles exhibited satisfying surface morphology, particle size, size distribution and stability. With an optimized theoretical drug loading (10\%), the drug-loaded PLGA nanoparticles exhibited negligible cytotoxicity against human oral squamous cell carcinoma cells. We noticed a biphasic drug release in vitro and the rate and cumulative release was higher in an acidic environment ( $\mathrm{pH} 4.5)$ compared to a neutral environment ( $\mathrm{pH}$ 7.4). The drug-loaded PLGA nanoparticles significantly inhibited the growth and lactic acid production of planktonic S. mutans as well as $S$. mutans biofilms. Our results indicate that the novel urea derivative-loaded PLGA nanoparticles serve as a promising anti-caries agent with remarkable pharmaceutical characteristics, low cytotoxicity, and satisfying antimicrobial effect.
\end{abstract}

Received 24th December 2021 Accepted 25th January 2022

DOI: 10.1039/d1ra09314b

rsc.li/rsc-advances bis[3,5-bis(trifluoromethyl)phenyl]urea (Fig. 1, CAS number: 3824-74-6), which exhibited a strong ability to increase fluoride toxicity on a cariogenic bacterial species, Streptococcus mutans (S. mutans). ${ }^{7}$ An investigation of the molecular structure showed that this chemical did not only interfere with fluoride transport but also had strong potential to exhibit antimicrobial effects alone. ${ }^{8}$ Previous studies have confirmed that similar urea derivatives can significantly inhibit the growth of Staphylococcus aureus and Pseudomonas aeruginosa. ${ }^{9}$ The diaryl rings contributed as hydrogen-bond donors and had the potential to bind and inhibit bacterial proteins such as DNA gyrase B and penicillin binding protein 1a (PBP 1a), which were known targets for several antibacterial agents. ${ }^{\mathbf{1 0 1 1}}$ Moreover, the trifluoromethyl groups on the diaryl rings can enhance membrane permeation of the drug and increase its lipophilicity and metabolic stability. ${ }^{12}$

While 1,3-bis[3,5-bis(trifluoromethyl)phenyl]urea has great potentials as a locally administered antimicrobial, its insolubility in water and difficulty of staying in oral cavity remains obstacles. Over the years, poly(lactic-co-glycolic acid) (PLGA)<smiles>O=C(Nc1cc(C(F)(F)F)cc(C(F)(F)F)c1)Nc1cc(C(F)(F)F)cc(C(F)(F)F)c1</smiles>

Fig. 1 1,3-Bis[3,5-bis(trifluoromethyl)phenyl]urea.

\footnotetext{
${ }^{a}$ Nanjing Stomatological Hospital, Medical School of Nanjing University, Nanjing, Jiangsu, 210008, China

${ }^{b}$ Department of Pediatric Dentistry, Nanjing Stomatological Hospital, Medical School of Nanjing University, Nanjing, Jiangsu, 210008, China

${ }^{c}$ Department of Oral Implantology, Nanjing Stomatological Hospital, Medical School of Nanjing University, Nanjing, Jiangsu, 210008, China. E-mail: 419311196@qq.com ${ }^{d}$ Department of Periodontology, Nanjing Stomatological Hospital, Medical School of Nanjing University, Nanjing, Jiangsu, 210008, China. E-mail: yanfh@nju.edu.cn

$\dagger$ These authors contributed equally to this study.
} 
nanoparticles, a FDA- and EMA-approved biodegradable drug delivery system, have been used as drug carriers for cancer, cardiovascular diseases, infections, and other health disorders. ${ }^{13,14}$ PLGA nanoparticulate systems can encapsulate various hydrophobic drugs and they have shown good stability with sustained drug release in vivo. ${ }^{15}$ The rate of polymer degradation has been found to be related to external $\mathrm{pH}$ conditions. Previous studies have reported that PLGA microspheres had an accelerated rate of degradation at lower $\mathrm{pH}$ compared to neutral state, ${ }^{16-18}$ indicating that drug release from PLGA systems could be $\mathrm{pH}$-responsive. It has been widely accepted that dental caries only occurs and progresses in acidic environments. $.^{19} \mathrm{~A} \mathrm{pH}-$ responsive drug carrier has long been favored in the development of anti-caries drugs. Therefore, PLGA is selected in the current study as an ideal carrier for 1,3-bis[3,5-bis(trifluoromethyl)phenyl]urea.

In this study, we loaded the novel urea derivative 1,3-bis [3,5-bis(trifluoromethyl)phenyl]urea in PLGA nanoparticles and explored its antimicrobial effects against cariogenic $S$. mutans. Nanoprecipitation was applied to obtain PLGA and drug-loaded PLGA nanoparticles, followed by physicochemical characterizations of nanoparticles, including examination of surface morphology, particle size, size distribution, polydispersity index (PDI), zeta potential, encapsulation efficiency, as well as loading efficiency. The stability and drug release profiles were evaluated under different $\mathrm{pH}$ conditions. Cytotoxicity of PLGA and drug-loaded PLGA nanoparticles was examined. Finally, the antibacterial efficacy of drug-loaded PLGA nanoparticles against planktonic $S$. mutans and $S$. mutans biofilms was determined.

\section{Materials and methods}

\section{Chemicals, test cells and bacteria, and growth conditions}

1,3-Bis[3,5-bis(trifluoromethyl)phenyl]urea was obtained from Sigma-Aldrich (St Louis, MO, USA). Poly(D,L-lactide-co-glycolide) (PLGA, lactide : glycolide 50:50, molecular weight $24000-$ $38000 \mathrm{Da})$ were obtained from Aladdin Biochemical Technology Co., Ltd (Shanghai, China). Chemicals including polysorbate 80 and acetonitrile which were used to prepare PLGA nanoparticles were purchased from Sigma-Aldrich and Titan Scientific Co., Ltd (Shanghai, China).

Oral squamous cell carcinoma (OSCC) cell line CAL27, provided by the American Type Culture Collection (ATCC; Manassas, VA, USA), was used for cytotoxicity test. Cells were grown in Dulbecco's modified Eagle medium, high glucose (DMEM-high, Gibco, NY, USA) containing $10 \%$ fetal bovine serum (FBS, Sigma-Aldrich) and 1\% penicillin-streptomycin solution (HyClone, Logan, USA) at $37^{\circ} \mathrm{C}$ with $5 \% \mathrm{CO}_{2}$.

S. mutans UA159 was used in this study for antimicrobial efficacy test. Bacterial strain was a gift from the State Key Laboratory of Oral Diseases (Sichuan University, Chengdu, China). For planktonic status, bacteria were cultured in Brain Heart Infusion (BHI, Becton, Dickinson and Company, USA) media and grown on $\mathrm{BHI}$ agar plates at $37{ }^{\circ} \mathrm{C}$ anaerobically $\left(10 \% \mathrm{H}_{2}, 5 \% \mathrm{CO}_{2}\right.$, and $\left.85 \% \mathrm{~N}_{2}\right)$. For $S$. mutans biofilms, BHIS (BHI medium containing $1 \%$ sucrose) was used. Biofilms were formed on the surface of clarified saliva-coated hydroxyapatite (sHA) discs. Clarified saliva was prepared by centrifuging human whole unstimulated saliva at $4000 \mathrm{rpm}$ for $20 \mathrm{~min}$ at $4{ }^{\circ} \mathrm{C}$, followed by filtration through $0.22 \mu \mathrm{m}$ polyethersulfone membrane..$^{20} \mathrm{HA}$ discs $(8 \mathrm{~mm}$ in diameter and $2 \mathrm{~mm}$ in thickness) were purchased from Baiameng Bioactive Materials Co., Ltd (Chengdu, China).

\section{Preparation of PLGA and drug-loaded PLGA nanoparticles}

The nanoprecipitation technology was used to prepare the PLGA and drug-loaded PLGA nanoparticles. Polysorbate 80 (w/v $0.1 \%$ ) was added in the aqueous phase. The organic phase was obtained by accurately weighing and dissolving $10 \mathrm{mg}$ PLGA and specified amount of 1,3-bis[3,5-bis(trifluoromethyl)phenyl] urea in $2 \mathrm{~mL}$ acetonitrile. The organic phase was added dropwise to $2 \mathrm{~mL}$ of the aqueous phase at a constant rate of 1 $\mathrm{mL} \min ^{-1}$ and thoroughly stirred at $1200 \mathrm{rpm}$ with a magnetic stirrer (big squid white, IKA, Germany). The theoretical drug loading of 1,3-bis[3,5-bis(trifluoromethyl)phenyl]urea in the PLGA was $100 \% \mathrm{w} / \mathrm{w}, 50 \% \mathrm{w} / \mathrm{w}, 20 \% \mathrm{w} / \mathrm{w}, 10 \% \mathrm{w} / \mathrm{w}$ and $5 \% \mathrm{w} / \mathrm{w}$, respectively. Acetonitrile was removed by evaporation in a ventilator cabinet at room temperature overnight. The next day, the suspension was filtered with $0.45 \mu \mathrm{m}$ filters (Titan Scientific, Shanghai, China) to remove the free drug. Finally, the resulting PLGA and drug-loaded PLGA nanoparticles suspension was directly used for further physicochemical characterization or stored at $4{ }^{\circ} \mathrm{C}$ until used for antimicrobial tests.

\section{Physicochemical characterization of nanoparticles}

Particle size and morphology. The morphology and size of nanoparticles were observed using Transmission Electron Microscope (TEM) (Tecnai G2 F20 S-TWIN; FEI Company, Hillsboro, OR, USA). A proper amount of prepared nanoparticles suspension was placed on the copper mesh and negatively stained with $0.5 \%$ phosphotungstic acid solution before observation. The particle size, size distribution, polydispersity index (PDI), and zeta potential of nanoparticles were measured by dynamic light scattering (DLS) using Zetasizer (Nano ZS90, Malvern, UK) at $25^{\circ} \mathrm{C}$ with an angle of detection of $180^{\circ}$. The fresh nanoparticles batches were suspended in deionized water (Millipore, USA) for analysis. Three independent samples were evaluated and results were expressed as mean value \pm standard deviation.

Stability of PLGA nanoparticles. To detect the stability of PLGA and drug-loaded nanoparticles, prepared nanoparticles were stored in different media, including citrate buffer $(\mathrm{pH} 4.5$ and $\mathrm{pH}$ 5.5, 0.01 M), phosphate buffered saline (pH 7.4, 0.01 M), and distilled water. The fresh nanoparticle batches were mixed with buffer solutions at a ratio of $1: 1$ and then placed in a water bath at $37^{\circ} \mathrm{C}$. At $0,3,9,24$ and $48 \mathrm{~h}$, samples were taken and examined with TEM and DLS to investigate the changes in particle distribution and size.

Determination of encapsulation efficiency and drug loading. The content of 1,3-bis[3,5-bis(trifluoromethyl)phenyl]urea in the PLGA nanoparticles was quantified by high performance liquid chromatography (HPLC, Agilent 1260 Infinity II, Agilent 
Technologies, Germany) with an Ultimate XB-C18 $(150 \mathrm{~mm} \times$ $4.6 \mathrm{~mm}, 5 \mu \mathrm{m}$ ) column. In the HPLC system, the mobile phase was methanol : water $(85: 15 \mathrm{v} / \mathrm{v})$ mixture and the isocratic flow

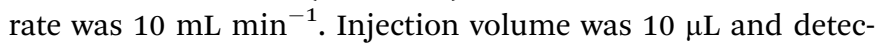
tion was performed at $260 \mathrm{~nm}$ wavelength. Briefly, a specific amount of drug-loaded PLGA nanoparticles were completely dissolved in HPLC-grade acetonitrile (Merck, USA) under magnetic stirring at a uniform speed of $1200 \mathrm{rpm}$ for $10 \mathrm{~min}$ to destroy the polymer network fully. The content of 1,3-bis[3,5bis(trifluoromethyl)phenyl]urea was then quantified with HPLC and the encapsulation and loading efficiency were determined using the following calculation formulas:

Encapsulation efficiency $(\% \mathrm{EE})=$ amount of drug in nanoparticles/total amount of drug added $\times 100 \%$.

Loading efficiency $(\%$ LE) $=$ amount of drug in nanoparticles/ total weight of nanoparticles $\times 100 \%$.

Release profiles under different $\mathbf{p H}$ conditions. The in vitro release profiles of 1,3-bis[3,5-bis(trifluoromethyl)phenyl]urea from PLGA nanoparticles under different $\mathrm{pH}$ conditions were assessed by determining the residual content of drug in the nanoparticles. A highly cariogenic $\mathrm{pH}$ (4.5) and a neutral $\mathrm{pH}$ (7.4) was selected to mimic intraoral conditions. Briefly, drugloaded nanoparticle suspensions were placed in either citrate buffer ( $\mathrm{pH} 4.5,0.01 \mathrm{M}$ ) or phosphate buffer ( $\mathrm{pH} 7.4,0.01 \mathrm{M})$. Suspensions were incubated at $37{ }^{\circ} \mathrm{C}$ and shaken horizontally at 160 strokes per min. At defined time points $(0,0.5,1,2,4,8$, $24,48,72,96,120,144$ and $168 \mathrm{~h}$ ), samples were taken and filtered to remove the released free drug. Samples were then dissolved in HPLC-grade acetonitrile and subsequently sent for HPLC to measure the content of 1,3-bis[3,5bis(trifluoromethyl)phenyl]urea using the same procedure described above. The content of the released drug was calculated by subtracting the residual drug quantity in nanoparticles at specific time points from the total drug quantity in nanoparticles at $0 \mathrm{~h}$. Cumulative release (\%) of drugs with time was also calculated.

\section{Antimicrobial activity of drug-loaded nanoparticles on $S$. mutans}

Inhibition on planktonic $\boldsymbol{S}$. mutans UA159. Antibacterial effects of drug-loaded nanoparticles on planktonic bacterial cells were examined with growth curve and lactic acid production. Overnight $S$. mutans UA159 cultures were inoculated into fresh BHI medium at a dilution of $1: 20$ and incubated at $37{ }^{\circ} \mathrm{C}$ anaerobically until $\mathrm{OD}_{600} \mathrm{~nm}$ reached 0.2 . Cells were then centrifuged and resuspended in fresh BHI medium. Twenty $\mu \mathrm{L}$ of PLGA nanoparticles $\left(5 \mathrm{mg} \mathrm{mL}^{-1}\right.$ ) or drug-loaded nanoparticles was added to $1.98 \mathrm{~mL}$ bacterial suspension and incubated at $37{ }^{\circ} \mathrm{C}$. Sterile water was added in the control group. Bacteria growth was monitored with Spectra MaxM3 (Molecular Devices, San Jose, CA, USA) by measuring $\mathrm{OD}_{600} \mathrm{~nm}$ every hour.

Lactic acid production of planktonic $S$. mutans cells in the presence of drug-loaded nanoparticles was measured as previously described. ${ }^{21}$ Briefly, $S$. mutans cells were incubated until mid-exponential phase $\left(\mathrm{OD}_{600 \mathrm{~nm}} \sim 0.5\right)$, harvested with centrifugation and resuspended in potassium phosphate buffer ( $\mathrm{pH} 7.0,90 \mathrm{mM})$. Either PLGA or drug-loaded PLGA nanoparticles were added and the suspensions were incubated at $37{ }^{\circ} \mathrm{C}$ for $1 \mathrm{~h}$. The same amount of sterile water was added as control. At the end of the incubation, the suspensions were centrifuged, and the supernatant was used to determine the amount of lactic acid enzymatically. ${ }^{22}$

Inhibition on $\boldsymbol{S}$. mutans biofilms

Preparation of the saliva coated-hydroxyapatite discs (s-HA). Hydroxyapatite (HA) discs were sonicated in deionized water for $10 \mathrm{~min}$ to remove free debris on the surface and then put in a glass Petri dish with deionized water for sterilization at $121{ }^{\circ} \mathrm{C}$ for $15 \mathrm{~min}$. The sterile HA discs were randomly placed into the wells of 24-well plates. A volume of $2 \mathrm{~mL}$ of clarified saliva (sterile saliva containing biologically active substances such as various proteins, inorganic ions and trace elements) was added to each well and incubated at $37{ }^{\circ} \mathrm{C}$ for $2 \mathrm{~h}$ to form saliva-coated hydroxyapatite discs (s-HA) as described previously. ${ }^{20}$

SEM images, CFU counts and $\mathrm{pH}$ measurement. Overnight cultures of $S$. mutans UA159 were transferred into fresh BHI medium until they reached the mid-exponential phase $\left(\mathrm{OD}_{600} \mathrm{~nm}\right.$ $\sim 0.5$ ) and were diluted $1: 100$ in fresh $\mathrm{BHI}$ with $1 \%$ sucrose (BHIS). Next, $1.98 \mathrm{~mL}$ of culture was transferred into wells of 24well plates with an sHA disc in each well. Twenty $\mu \mathrm{L}$ suspensions

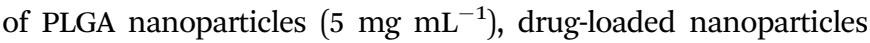
(theoretical drug loading $10 \% \mathrm{w} / \mathrm{w}$ ), sodium fluoride (final concentration $8 \mathrm{mM}$ ) or sterile water (control) was added in each well. Cultures were then incubated at $37{ }^{\circ} \mathrm{C}$ anaerobically and media were changed every $24 \mathrm{~h}$. Biofilms formed at $24 \mathrm{~h}$ were examined with scanning electron microscopy (SEM) (Quanta 400 FEG, FEI, USA). Samples were taken and washed twice with CPW and then fixed in $2.5 \%$ glutaraldehyde at $4{ }^{\circ} \mathrm{C}$ overnight. They were then serially dehydrated with ethanol $(50 \%, 60 \%, 70 \%, 80 \%, 90 \%$, and $100 \%$ ). Photos were examined at $\times 1000, \times 5000$, and $\times 20000$ magnification under the microscope with an accelerating voltage of $20 \mathrm{kV}$. The images displayed are randomly selected from at least three fields.

After 24,48 and $72 \mathrm{~h}$ incubation at $37^{\circ} \mathrm{C}$ anaerobically, the HA discs were washed twice with sterile cysteine peptone water (CPW, containing yeast extract, peptone, sodium chloride, and cysteine $\mathrm{HCl}, \mathrm{pH}$ 7.2) to remove loosely attached bacteria. Then, the discs were transferred to a $5 \mathrm{~mL}$ tube with $2 \mathrm{~mL}$ sterile CPW, and the $S$. mutans biofilms were dispersed by cell scrapers and sonicated on ice for $10 \mathrm{~min}$ at an amplitude of $40 \mathrm{~W}$. The bacterial suspension was serially diluted with sterile deionized water, and $100 \mu \mathrm{L}$ of the diluted solution was plated on BHI agar plates, incubating at $37{ }^{\circ} \mathrm{C}$ under anaerobic conditions for $48 \mathrm{~h}$ before the colony forming unit (CFU) was counted.

The supernatant (centrifuged at $5000 \mathrm{rpm}$ for $10 \mathrm{~min}$ at $4{ }^{\circ} \mathrm{C}$ ) of biofilms at 24, 48 and $72 \mathrm{~h}$ were taken and $\mathrm{pH}$ was measured with $\mathrm{pH}$ meter (FiveEasy Plus, FE28-standard, Mettler Toledo, Switzerland).

Bacteria live/dead staining assay. Confocal laser scanning microscopy (CLSM, Nikon A1; Nikon Inc., Tokyo, Japan) was used to visualize live and dead bacterial cells in $24 \mathrm{~h}$ biofilms. 
Samples were first washed with $0.9 \%$ sodium chloride to remove loosely adhered cells. LIVE/DEAD BacLight Bacterial Viability Kit (Life Technologies Corporation, New York, USA) was used according to the manufacturer's instructions. SYTO 9 (excitation, $480 \mathrm{~nm}$; emission, $500 \mathrm{~nm}$ ) and propidium iodide (PI, excitation, $490 \mathrm{~nm}$; emission, $635 \mathrm{~nm}$ ) were used to stain viable and dead cells, respectively. Biofilm images were captured with CLSM at magnifications of $10 \times$. The biofilm depth was examined by generating the optical sections of $10 \mu \mathrm{m} .^{23}$ ImageJ software (v1.48, National Institutes of Health, USA) was used to quantify the integrated density. The quantity of viable and dead cells was represented by the integrated density of green and red fluorescence, respectively.

\section{Cytotoxicity assay}

The cytotoxicity of PLGA and drug-load PLGA nanoparticles was detected using OSCC CAL27 cell line with CCK-8 assay kit (Meilun Biotechnology, Dalian, China). In brief, 5000 cells per well were seeded in $100 \mu \mathrm{L}$ of growth medium (high-glucose Dulbecco's modified Eagle's medium (DMEM) with 10\% (v/v) fetal bovine serum and $1 \%(\mathrm{v} / \mathrm{v})$ penicillin-streptomycin solution.) in 96-well polypropylene microtiter plates and then cultured in an incubator at $37{ }^{\circ} \mathrm{C}$ with $5 \% \mathrm{CO}_{2}$ for $24 \mathrm{~h}$. After the cells adhered to the wall, the culture medium was replaced by a fresh medium containing nanoparticles of different concentrations. Control groups (with deionized water) and blank groups (cell-free medium) were set. Five replicates were placed for each concentration. After incubation for 24, 48 and $72 \mathrm{~h}, 10$ $\mu \mathrm{L}$ CCK-8 solution was added to each well and incubated at $37^{\circ} \mathrm{C}$ for $2 \mathrm{~h}$. Finally, the light absorption value of each well was detected by Spectra Max M3 at $450 \mathrm{~nm}$.

\section{Results}

\section{The morphology and size of nanoparticles}

The PLGA and drug-loaded PLGA nanoparticles were successfully prepared by nanoprecipitation technology. TEM images showed that the nanoparticles were almost spherical in shape and had a smooth surface without pore. They were dispersed with each other (Fig. 2A and B). The size of all nanoparticles was less than $200 \mathrm{~nm}$, which was confirmed by DLS analysis (Fig. 2C and D). Also, DLS showed that the nanoparticles had a narrow and normalized size distribution.

\section{Encapsulation and loading efficiency of drug-loaded PLGA nanoparticles}

Encapsulation efficiency (\% EE) and loading efficiency (\% LE) of drug-loaded PLGA nanoparticles were evaluated with different theoretical drug loadings. Particle size, PDI, and zeta potential were also measured. Table 1 showed that the average particle size of PLGA nanoparticles was $102.63 \pm 1.46 \mathrm{~nm}$, which was significantly smaller than that of drug-loaded PLGA nanoparticles (ranging from $112.6 \mathrm{~nm}$ to $140.6 \mathrm{~nm}$ ). Particle sizes increased with the rise of theoretical drug loading. Zeta potentials of nanoparticles also increased in negativity with the increase of theoretical drug loading (from $-13.1 \mathrm{mV}$ to -27.3 $\mathrm{mV}$ ). PDIs of all groups were less than 0.2 , indicating a narrow size distribution. Based on \% EE and \% LE of different theoretical drug loading, $10 \% \mathrm{w} / \mathrm{w}$ was selected for further experiments as it exhibited a satisfying \% EE $(94.8 \pm 2.8 \%)$ and \% LE $(8.7 \pm 0.2 \%)$.

\section{Stability of PLGA and drug-loaded PLGA nanoparticles}

Fig. 3 shows the change of particle sizes of PLGA and drugloaded PLGA nanoparticles at $37{ }^{\circ} \mathrm{C}$ under different $\mathrm{pH}$
A
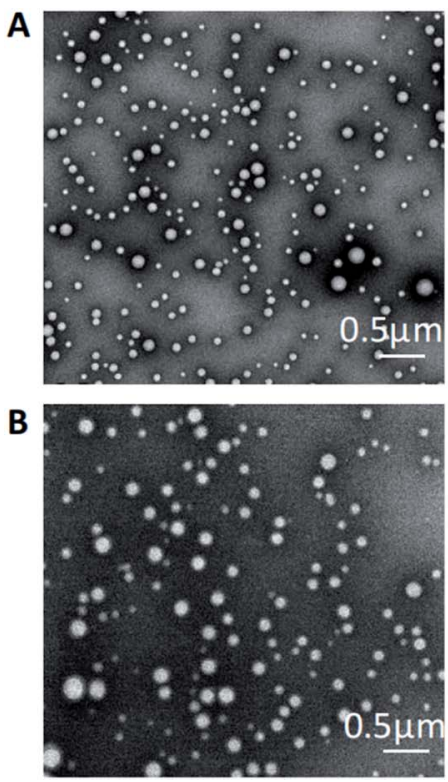
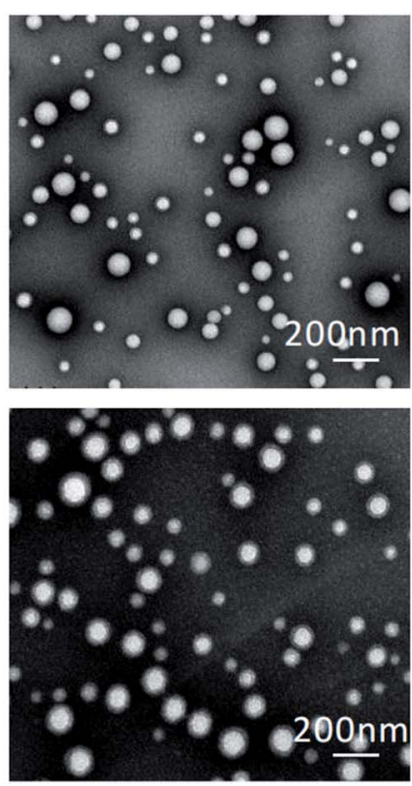

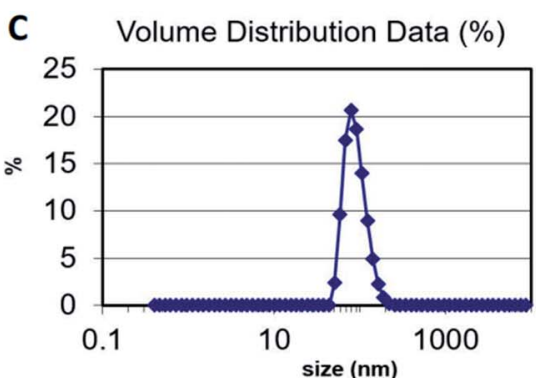

D

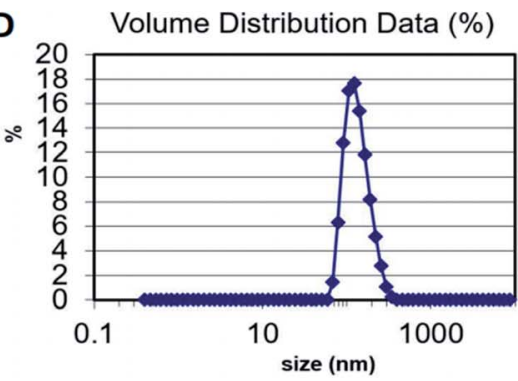

Fig. 2 Representative TEM images of (A) PLGA nanoparticles and (B) drug-loaded PLGA nanoparticles. DLS size distribution of (C) PLGA nanoparticles and (D) drug-loaded PLGA nanoparticles. 
Table 1 Physicochemical characterizations of drug-loaded PLGA nanoparticles with different theoretical drug loadings. Means \pm standard deviations from three independent experiments are presented

\begin{tabular}{lllll}
\hline $\begin{array}{l}\text { Theoretical drug } \\
\text { loading }(\%, w / w)\end{array}$ & Particle size $(\mathrm{nm})$ & PDI & Zeta potential $(\mathrm{mV})$ & $\% \mathrm{EE}$ \\
\hline 0 & $102.6 \pm 1.5$ & $0.07 \pm 0.04$ & $-13.1 \pm 0.4$ & - \\
100 & $140.7 \pm 0.6$ & $0.08 \pm 0.04$ & $-13.3 \pm 0.8$ & $11.5 \pm 0.7$ \\
50 & $127.9 \pm 2.2$ & $0.11 \pm 0.02$ & $-14.2 \pm 1.1$ & $40.9 \pm 9.6$ \\
20 & $120.2 \pm 0.6$ & $0.15 \pm 0.06$ & $-24.9 \pm 1.0$ & $64.6 \pm 14.5$ \\
10 & $113.5 \pm 2.1$ & $0.04 \pm 0.01$ & $-25.6 \pm 0.5$ & $16.8 \pm 2.9$ \\
5 & $112.6 \pm 2.0$ & $0.05 \pm 0.02$ & $-27.3 \pm 0.7$ & $94.8 \pm 2.8$ \\
& & & $95.6 \pm 1.5$
\end{tabular}
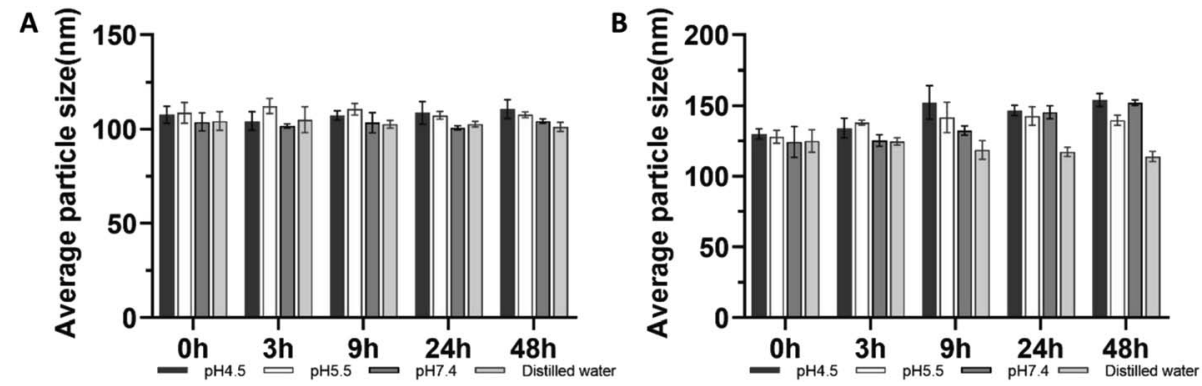

Fig. 3 Stability of (A) PLGA and (B) drug-loaded PLGA nanoparticles. Drug-loaded PLGA nanoparticles with 10\% theoretical loading was used. Means \pm standard deviations from three independent experiments are presented.

conditions during $48 \mathrm{~h}$ incubation. Changes were insignificant, while we noticed the size of drug-loaded particles had a slight trend of increasing under acidic conditions. Otherwise, particle sizes remained around $100 \mathrm{~nm}$ for PLGA nanoparticles and $130 \mathrm{~nm}$ for drug-loaded nanoparticles throughout the incubation period.

\section{In vitro drug release behavior of drug-loaded PLGA nanoparticles}

The release behavior of 1,3-bis[3,5-bis(trifluoromethyl)phenyl] urea from drug-loaded PLGA was evaluated at different $\mathrm{pH}$ values (7.4 and 4.5). The result is presented in Fig. 4. At pH 4.5, approximately $45 \%$ of the total loaded content was released from drug-loaded PLGA during the first $24 \mathrm{~h}$. After the initial rapid release, drug release continued and finally reached $61 \%$ of the total loaded content after 7 days. In neutral environments, a relatively smaller amount of drug $(33 \%$ of total loaded content) was released within the first $24 \mathrm{~h}$, and the cumulative release was about $46 \%$ of total loaded content after 7 days.

\section{Inhibition on growth and lactic acid production in planktonic} S. mutans

The growth profiles of $S$. mutans UA159 in the presence of PLGA and drug-loaded nanoparticles with different theoretical loadings were shown in Fig. 5A. PLGA nanoparticles did not inhibit bacterial growth. All drug-loaded nanoparticles showed almost complete growth inhibition. The inhibitive effect was similar to that of $8 \mathrm{mM} \mathrm{NaF}$.

Fig. 5B exhibited lactic acid production profiles of $S$. mutans UA159 treated with PLGA nanoparticles for $1 \mathrm{~h}$. The co-

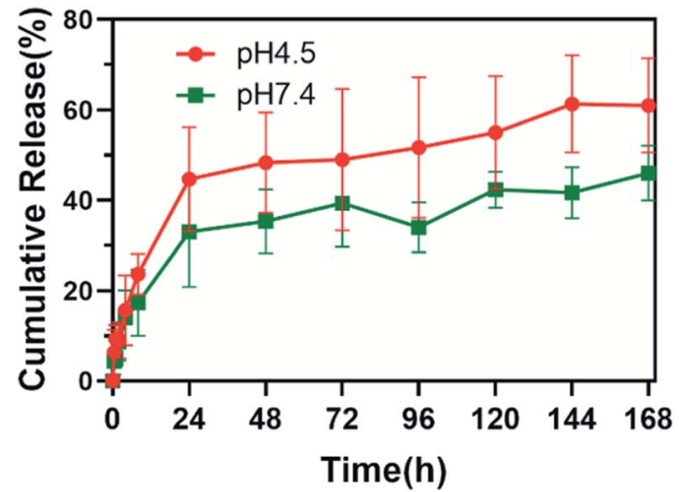

Fig. 4 In vitro release of 1,3-bis[3,5-bis(trifluoromethyl)phenyl]urea from drug-loaded PLGA nanoparticles under different $\mathrm{pH}$ conditions. Means \pm standard deviations from three independent experiments are presented.

incubation of bacterial cells with PLGA nanoparticles did not affect the ability of lactic acid production $(1.16 \pm 0.21 \mathrm{mM})$. Drug-loaded nanoparticles with $10 \%$ theoretical loading led to significantly less production of lactic acid $(0.70 \pm 0.25 \mathrm{mM})$.

\section{Inhibition on formation and acid production of $S$. mutans biofilm}

SEM images confirmed that drug-loaded PLGA nanoparticles inhibited the formation and development of biofilms on sHA discs (Fig. 6). While we noticed a thick layer of biofilms as well as a vast amount of extracellular matrix in control and PLGA 
A

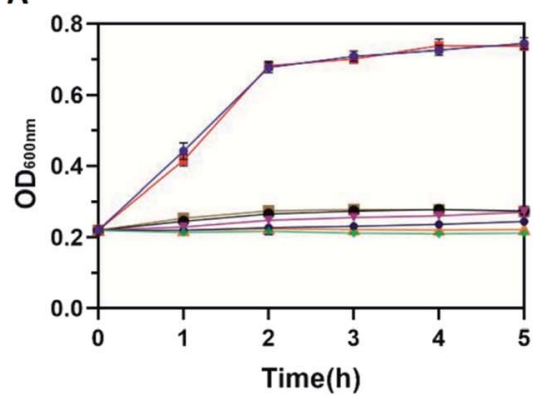

B

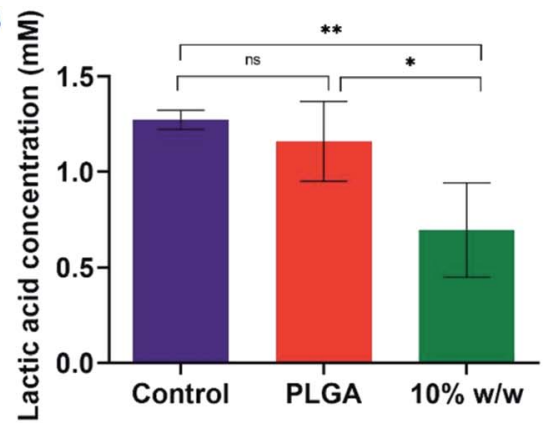

Fig. 5 (A) Growth curve and (B) lactic acid production of S. mutans UA159 with drug-loaded PLGA nanoparticles. W/w indicated the theoretical drug loading of 1,3-bis[3,5-bis(trifluoromethyl)phenyl]urea in PLGA nanoparticles. This experiment was repeated three times and means \pm standard deviations are presented. * indicates $p<0.05, * *$ indicates $p<0.01$.

groups, biofilms appeared thinner and less matrix was visible in drug-loaded PLGA group and sodium fluoride group (Fig. 6A).

CFU counts confirmed that the presence of drug-loaded PLGA nanoparticles significantly decreased the number of viable cells in 24,48 and $72 \mathrm{~h}$ biofilms ( $* * p<0.01$, Fig. $6 \mathrm{~B})$. In control and PLGA groups, CFU counts reached $1.6 \times 10^{9}, 1.8 \times$ $10^{10}$ and $1.9 \times 10^{11} \mathrm{CFU} \mathrm{mL}^{-1}$ at 24,48 and $72 \mathrm{~h}$ respectively. In drug-loaded PLGA group, CFU counts was $1.7 \times 10^{7}, 2.0 \times 10^{7}$
A
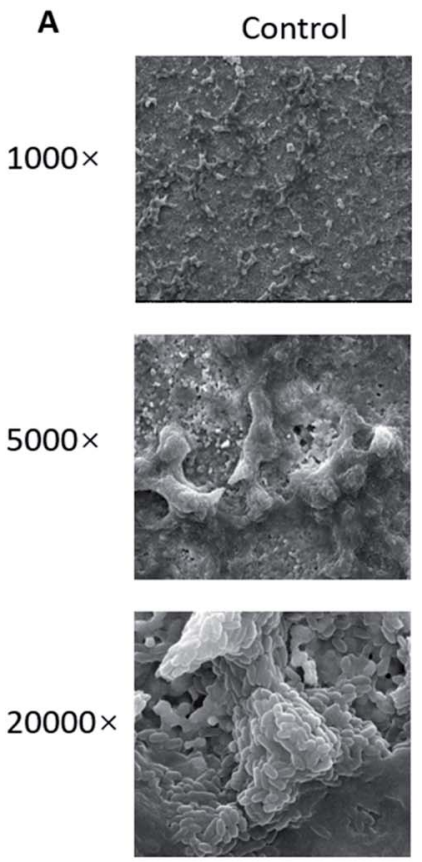

$5000 \times$

$1000 \times$

B

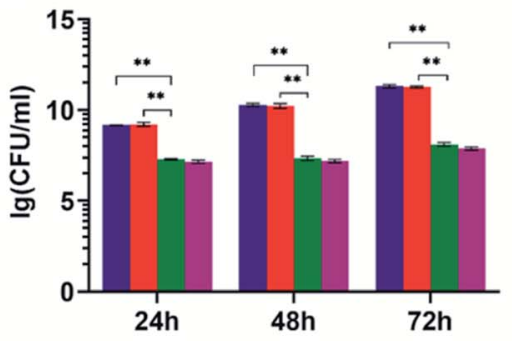

PLGA
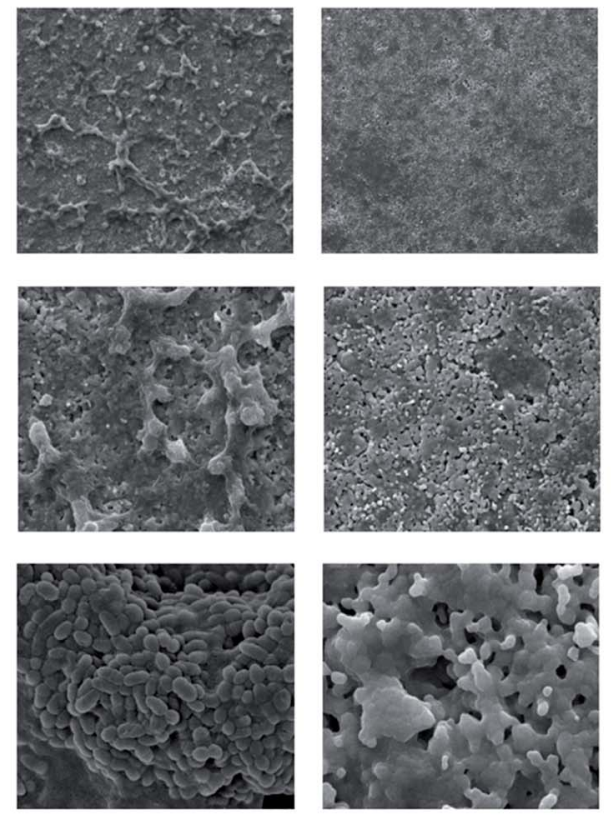

C

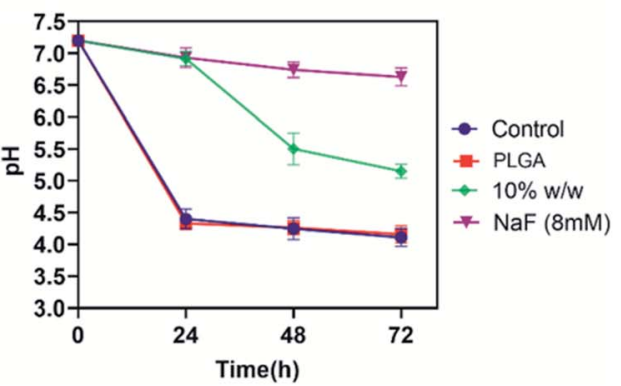

Fig. 6 (A) Representative SEM images of 24 h S. mutans biofilms in different groups. Photos were taken at $1000 \times, 5000 \times$ and $20000 \times$ magnification. (B) CFU counts of S. mutans biofilms. Data were collected at 24,48 and $72 \mathrm{~h}$. (C) pH of supernatant of biofilms. Drug-loaded PLGA nanoparticles with $10 \%$ theoretical loading was used. This experiment was repeated three times and means \pm SD are presented. $* *$ indicates $p<0.01$. 
and $1.1 \times 10^{8} \mathrm{CFU} \mathrm{mL} \mathrm{mL}^{-1}$ at 24,48 and $72 \mathrm{~h}$ respectively. No significant difference was observed between drug-loaded PLGA and sodium fluoride groups.

At different time points (24, 48 and $72 \mathrm{~h}$ ), $\mathrm{pH}$ of supernatant of biofilms was determined to reflect the ability of the biofilms to produce acid. As shown in Fig. 6C, biofilms in control and PLGA groups had similar supernatant $\mathrm{pH}$, which dropped to $\mathrm{pH}$ 4.5 in the first $24 \mathrm{~h}$. Drug-loaded PLGA nanoparticles significantly alleviated the $\mathrm{pH}$ drop, especially in the first $24 \mathrm{~h}$. During the first $24 \mathrm{~h}, \mathrm{pH}$ dropped from 7.2 to around 6.9. The decrease was similar to the NaF group during this period. The $\mathrm{pH}$ drop became more obvious afterwards. By the end of $72 \mathrm{~h}$, the $\mathrm{pH}$ of the drug-loaded PLGA group was about 5.2 (Fig. 6C).

\section{Live/dead staining of biofilms}

As illustrated in Fig. 7A, CLSM images showed that the treatment of drug-loaded PLGA nanoparticles significantly increased fluorescence of dead bacterial cells in $24 \mathrm{~h}$ biofilms. The quantified integrated intensity of green and red fluorescence showed that the calculated live/dead bacterial ratio of $24 \mathrm{~h} S$. mutans biofilms exposed to the drug-loaded PLGA nanoparticles was remarkably lower than that of the control group and PLGA group. Furthermore, differences were noticed in the thickness of $24 \mathrm{~h} S$. mutans biofilms. The thickness of biofilms treated by sterile water and PLGA nanoparticles for $24 \mathrm{~h}$ was similar (approximately $90 \mu \mathrm{m}$ ), which was thicker than that of the biofilms treated by the drug-loaded PLGA nanoparticles samples (approximately $50 \mu \mathrm{m}$, Fig. 7B).

\section{Cytotoxicity assay}

The cell cytotoxicity was evaluated with OSCC CAL-27. The viability of cells was tested 24,48 and $72 \mathrm{~h}$ after the cells were treated with negative control, PLGA and drug-loaded PLGA (theoretical drug loading $10 \% \mathrm{w} / \mathrm{w}$ ). Fig. 8 showed that both PLGA and drug-loaded PLGA nanoparticles did not affect the viability of cells significantly. The cell viability of CAL27 cells after co-incubation with drug-loaded PLGA nanoparticles for 24 $(99.3 \% \pm 4.5 \%), 48(96.2 \% \pm 9.8 \%)$ and $72(96.2 \% \pm 3.8 \%) \mathrm{h}$ was higher than $90 \%$.

\section{Discussion}

The emergence of antimicrobial resistance in oral bacteria has been a rising problem when developing caries-preventive agents. In order to avoid drug resistance and promote effectiveness in local environment, small molecule antimicrobials have been of interest. This current study encapsulated a novel urea derivative, 1,3-bis[3,5-bis(trifluoromethyl)phenyl]urea, in biodegradable PLGA nanoparticles. The drug-loaded PLGA nanoparticles exhibited satisfying physicochemical

A

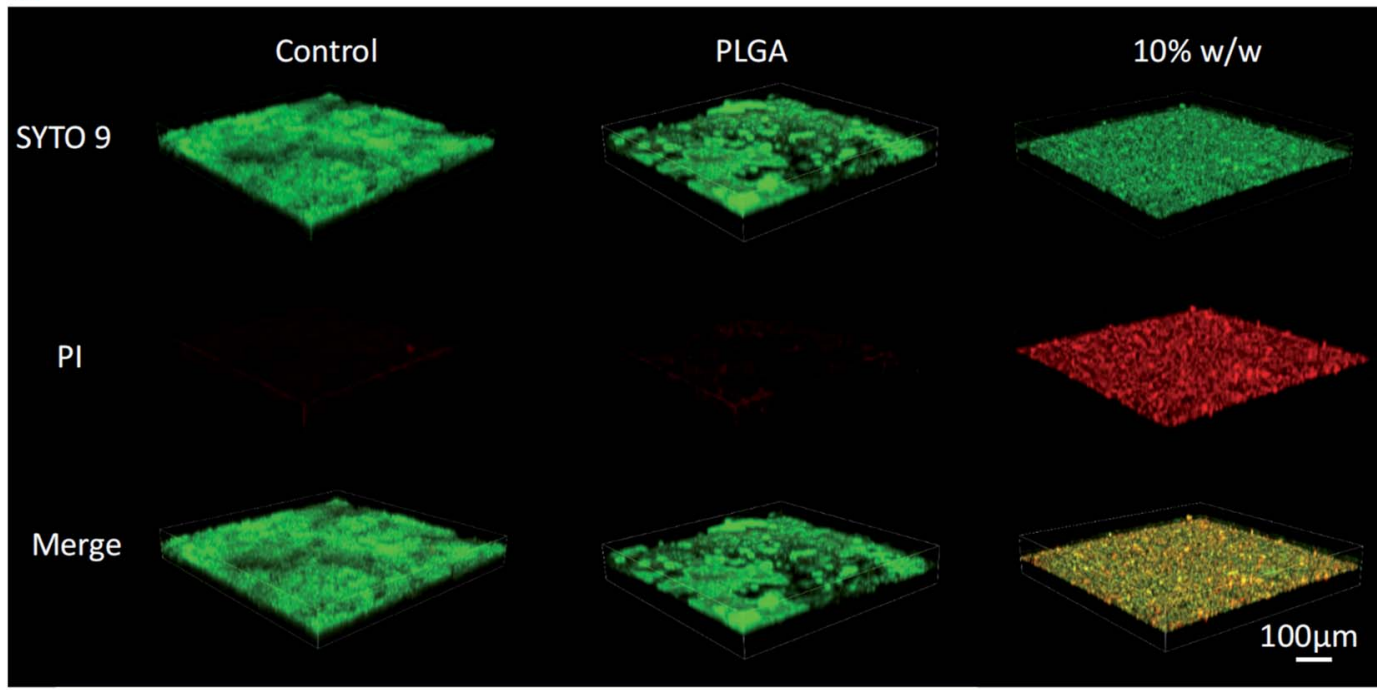

B
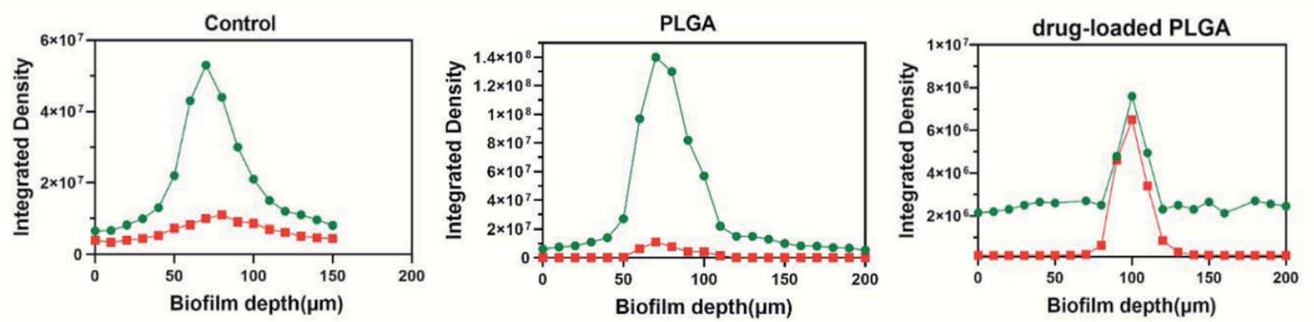

Fig. 7 (A) Representative CLSM scans of 24 h S. mutans biofilms stained with live/dead staining in different groups. Three-dimensional images were constructed by Nikon Capture NX2 software. Viable cells are shown in green and dead cells are shown in red. Yellow pixels represent the overlap of viable and dead bacterial cells. (B) The integrated density of $24 \mathrm{~h} \mathrm{~S}$. mutans biofilms stained in green and red fluorescence from the deepest layer $(0 \mu \mathrm{m})$ to the outer layer at $10 \mu \mathrm{m}$ intervals. Drug-loaded PLGA nanoparticles with 10\% theoretical loading was used. 


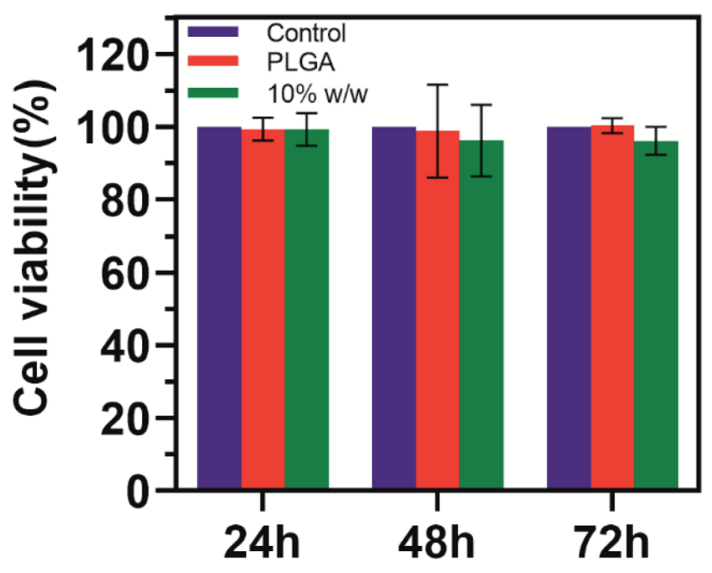

Fig. 8 Cytotoxicity assay of PLGA and drug-loaded PLGA nanoparticles. Drug-loaded PLGA nanoparticles with 10\% theoretical loading was used. This experiment was repeated three times and means \pm standard deviations are represented.

characterizations and acceptable cytotoxicity. Moreover, the antimicrobial effect of the drug-loaded PLGA nanoparticles was confirmed in planktonic $S$. mutans as well as $S$. mutans biofilms. This novel urea derivative-loaded PLGA nanoparticles provide as a promising agent to be used in caries prevention and treatment.

The application of urea derivatives as antimicrobials could date back to the 1940s when the antimicrobial activity of urea derivatives against Gram-positive bacteria was first reported by Weinstein L. et $a .^{24}$ In the past several years, different urea derivates have been developed to target bacteria including Escherichia coli, Staphylococcus aureus, and Pseudomonas aeruginosa. 1,3-Bis[3,5-bis(trifluoromethyl)phenyl]urea was first identified as a fluoride toxicity agonist due to its strong ability to bind $\mathrm{F}^{-}$mediated by the diaryl rings. ${ }^{7,25}$ We identified several functional groups in its chemical structure and realized that it had the potential to exert antimicrobial effect alone (as described in Introduction). In order to deliver and confirm the antimicrobial effect, PLGA nanoparticles were employed.

The drug-loaded PLGA nanoparticles exhibited satisfying pharmaceutical characters. The particle size is a crucial parameter as it directly affects the physical stability, cellular uptake, biodistribution, and drug release. ${ }^{26}$ The average size of the prepared nanoparticles was 100-140 nm, which was within the ideal range of nanoparticles for drug delivery. ${ }^{27}$ The nanoparticle size increased with the rise of theoretical drug loading, which could be the result of the increased number of active ingredients encapsulated in nanoparticles. Also, the PDI values of all prepared nanoparticles $(0.04-0.15)$ indicated that the nanoparticles were monodispersed, ${ }^{28}$ as observed in TEM images (Fig. 2). Zeta potentials of prepared nanoparticles represented carboxyl groups on the surface of the PLGA polymers and have been previously reported to be associated with the stability of nanoparticles in colloidal systems. ${ }^{29}$ All nanoparticles prepared in this study had negative zeta potentials, ranging from -13.1 to $-27.3 \mathrm{mV}$, indicating that they were relatively stable. This was further confirmed with the stability test (Fig. 3). By optimizing theoretical loading, we reached a high \% EE and \% LE (94.8\% and 8.7\% respectively).

The in vitro release profiles of 1,3-bis[3,5-bis(trifluoromethyl) phenyl] urea from the drug-loaded nanoparticles in different $\mathrm{pH}$ environments showed a biphasic configuration, consisting of an initial burst followed by a steady, sustained release (Fig. 4). This was in line with previous studies which also used PLGA as drug carriers. ${ }^{30}$ During the first 24 hours, the burst release could be related to the free drug which was absorbed on the surface of the PLGA nanoparticles. After the initial burst release, the profile showed a steady sustained-release manner for over $168 \mathrm{~h}$, probably due to the drug encapsulated at the core compartment retained firmly inside the nanoparticle. ${ }^{31}$ There are also reports that the encapsulated drug released slowly because of the relatively dense polymer or the few existing pores during the degradation and hydration proceed of PLGA nanoparticles. $^{32,33}$ It is worth mentioning that the release profile of the drug from PLGA nanoparticles was $\mathrm{pH}$-responsive. The rate and cumulative release of drug were significantly higher in acidic environment compared to neutral environment. This was closely related to the $\mathrm{pH}$-responsiveness of PLGA polymer. Low $\mathrm{pH}$ condition catalyzes the breakage of ester bond of polymerization and enhances erosion of PLGA, thus accelerating drug release. ${ }^{34}$ Specifically, the $\mathrm{pH}$-dependent release is highly appreciated for caries prevention, as only under acidic conditions does the disease initiate and progress.

In vitro antimicrobial experiments showed that drug-loaded PLGA nanoparticles inhibited the growth and acid production of both planktonic $S$. mutans and $S$. mutans biofilms. The ability to produce acid and form biofilms are the major virulence factors of $S$. mutans. ${ }^{35}$ It is somewhat surprising that the novel urea derivative used in this study can inhibit both processes. As described previously, in silico studies have predicted that similar urea derivatives can bind and inhibit key enzymes such as DNA gyrase B and PBP. ${ }^{36,37}$ The binding seems to be mediated by hydrogen bond between enzymes and the diaryl rings in the drug. It is speculated that 1,3-bis[3,5-bis(trifluoromethyl) phenyl]urea could also bind to other enzymes and act as an inhibitor, especially enzymes associated with metabolic pathways and biofilm formation. For example, we noticed that the extracellular matrix in biofilms treated with drug-loaded PLGA nanoparticles was significantly less with SEM examination (Fig. 6A). The activity of glucosyltransferase, which is responsible for the production of extracellular polysaccharides (EPS), was highly likely to be inhibited. Molecular docking and in vitro examinations will be performed to further confirm the binding of specific proteins with 1,3-bis[3,5-bis(trifluoromethyl)phenyl] urea. In addition, the urea derivative could be hydrolyzed by urease to produce $\mathrm{NH}_{3}$, which is an important alkalic substance in oral cavity and can be related to the limited $\mathrm{pH}$ drop in biofilms. $^{38}$

\section{Conclusion}

In conclusion, by encapsulating 1,3-bis[3,5-bis(trifluoromethyl) phenyl]urea in PLGA nanoparticles, we aimed to develop a novel anti-caries agent. The drug is released from the nanoparticles in 
a pH-dependent and slow released manner. Both acid production and biofilm formation of $S$. mutans are inhibited by the drug-loaded nanoparticles. Furthermore, the mechanism of the novel anti-caries agent to inhibit $S$. mutans still requires further research.

\section{Conflicts of interest}

There are no conflicts to declare.

\section{Acknowledgements}

This work was supported by the National Natural Science Funds (grant number 82001035); the Jiangsu Natural Science Funds (grant number SBK2020041847); and the Nanjing Clinical Research Center for Oral Diseases (grant number 2019060009).

\section{References}

1 J. Y. Niu, I. X. Yin, W. K. K. Wu, Q. L. Li, M. L. Mei and C. H. Chu, Arch. Oral Biol., 2021, 122, 105022.

2 W. Qiu, Y. Zhou, Z. Li, T. Huang, Y. Xiao, L. Cheng, X. Peng, L. Zhang and B. Ren, BioMed Res. Int., 2020, 2020, 5658212.

3 Y. Liao, J. Chen, B. W. Brandt, Y. Zhu, J. Li, C. van Loveren and D. M. Deng, PLoS One, 2015, 10, e0122630.

4 T. M. Karpinski and A. K. Szkaradkiewicz, Eur. Rev. Med. Pharmacol. Sci., 2015, 19, 1321-1326.

5 L. Drew, Nature, 2016, 537, S60-S62.

6 Q. Zhang, B. Nijampatnam, Z. Hua, T. Nguyen, J. Zou, X. Cai, S. M. Michalek, S. E. Velu and H. Wu, Sci. Rep., 2017, 7, 5974.

7 J. W. Nelson, M. S. Plummer, K. F. Blount, T. D. Ames and R. R. Breaker, Chem. Biol., 2015, 22, 527-534.

8 Y. Fan, C. Payne and S. R. Kass, J. Org. Chem., 2018, 83, 10855-10863.

9 Y. L. Wu, Yaохие Хиеbao, 1965, 12, 523-532.

10 C. Limban, M. C. Chifiriuc, M. T. Caproiu, F. Dumitrascu, M. Ferbinteanu, L. Pintilie, A. Stefaniu, I. M. Vlad, C. Bleotu, L. G. Marutescu and D. C. Nuta, Molecules, 2020, 25, DOI: 10.3390/molecules25071478.

11 F. M. Sroor, A. M. Othman, M. A. Tantawy, K. F. Mahrous and M. E. El-Naggar, Bioorg. Chem., 2021, 112, 104953.

12 J. A. Ma and D. Cahard, Chem. Rev., 2008, 108, PR1-PR43.

13 M. Mir, N. Ahmed and A. U. Rehman, Colloids Surf., B, 2017, 159, 217-231.

14 F. Danhier, E. Ansorena, J. M. Silva, R. Coco, A. Le Breton and V. Preat, J. Controlled Release, 2012, 161, 505-522.

15 M. Dandamudi, P. McLoughlin, G. Behl, S. Rani, L. Coffey, A. Chauhan, D. Kent and L. Fitzhenry, Pharmaceutics, 2021, 13, DOI: 10.3390/pharmaceutics13101590.

16 B. S. Zolnik and D. J. Burgess, J. Controlled Release, 2007, 122, 338-344.
17 Y. Hua, Y. Su, H. Zhang, N. Liu, Z. Wang, X. Gao, J. Gao and A. Zheng, Drug Delivery, 2021, 28, 1342-1355.

18 Y. Liu, A. H. Ghassemi, W. E. Hennink and S. P. Schwendeman, Biomaterials, 2012, 33, 7584-7593.

19 W. Krzysciak, A. Jurczak, D. Koscielniak, B. Bystrowska and A. Skalniak, Eur. J. Clin. Microbiol. Infect. Dis., 2014, 33, 499-515.

20 X. Wang, L. Liu, X. Zhou, Y. Huo, J. Gao and H. Gu, BMC Oral Health, 2020, 20, 169.

21 Y. Liao, B. W. Brandt, M. Zhang, J. Li, W. Crielaard, C. van Loveren and D. M. Deng, Antimicrob. Agents Chemother., 2016, 60, 7509-7512.

22 W. A. Belli, D. H. Buckley and R. E. Marquis, Can. J. Microbiol., 1995, 41, 785-791.

23 M. Vaknin, D. Steinberg, J. D. Featherstone and O. Feuerstein, Laser Med. Sci., 2020, 35, 709-718.

24 L. Weinstein and A. McDonald, Science, 1945, 101, 44-45.

25 N. Busschaert and P. A. Gale, Angew. Chem., Int. Ed., 2013, 52, 1374-1382.

26 G. Mittal, D. K. Sahana, V. Bhardwaj and M. N. Ravi Kumar, J. Controlled Release, 2007, 119, 77-85.

27 W. Huang and C. Zhang, Biotechnol. J., 2018, 13, DOI: 10.1002/biot.201700203.

28 A. A. Öztürk, L. M. Banderas, M. D. C. Otero, E. Yenilmez, B. Şenel and Y. Yazan, Trop. J. Pharm. Res., 2019, 18, DOI: 10.4314/tjpr.v18i1.1.

29 Z. Rahman, A. S. Zidan, M. J. Habib and M. A. Khan, Int. J. Pharm., 2010, 389, 186-194.

30 Y. Wang and Y. Tan, J. Drug Delivery Sci. Technol., 2016, 36, 183-191.

31 C. J. Martinez Rivas, M. Tarhini, W. Badri, K. Miladi, H. Greige-Gerges, Q. A. Nazari, S. A. Galindo Rodriguez, R. A. Roman, H. Fessi and A. Elaissari, Int. J. Pharm., 2017, 532, 66-81.

32 S. Fredenberg, M. Wahlgren, M. Reslow and A. Axelsson, Int. J. Pharm., 2011, 415, 34-52.

33 J. Wang, B. M. Wang and S. P. Schwendeman, J. Controlled Release, 2002, 82, 289-307.

34 N. Faisant, J. Siepmann and J. P. Benoit, Eur. J. Pharm. Sci., 2002, 15, 355-366.

35 L. Jia, S. Tao, J. Yang, K. Liang, Z. Yu, Y. Gao, M. Fan, M. Zhang, L. He and J. Li, Colloids Surf., B, 2021, 197, 111409.

36 K. W. Pugh, Z. Zhang, J. Wang, X. Xu, V. Munthali, A. Zuo and B. S. J. Blagg, ACS Med. Chem. Lett., 2020, 11, 1535-1538.

37 Z. T. Wen, J. P. Bitoun and S. Liao, PLoS One, 2015, 10, e0124319.

38 Y. L. Liu, M. Nascimento and R. A. Burne, Int. J. Oral Sci., 2012, 4, 135-140. 